\title{
Primordial binary evolution and blue stragglers
}

\author{
Xuefei Chen and Zhanwen Han \\ National Astronomical Observatories/Yunnan Observatory, Chinese Aacademy of Sciences, \\ Kunming, 650011, P. R. China \\ email: [xuefeichen717, zhanwenhan] @hotmail.com
}

\begin{abstract}
Blue stragglers have been found in all populations. They are important in both stellar evolution and stellar population synthesis. Much evidence shows that blue stragglers are relevant to primordial binaries. Here, we summarize the links between binary evolution and blue stragglers, describe the characteristics of blue stragglers originating from different binary evolutionary channels and show their consequences for binary population synthesis, such as for the integrated spectral-energy distribution, the colour-magnitude diagram, their specific frequency, and their influence on colours, etc.
\end{abstract}

Keywords. binaries: close, stars: evolution, blue stragglers

\section{Introduction}

Blue stragglers (BSs) are an important population component in stellar evolution as well as in star clusters. These objects have remained on the main sequence for a time exceeding that expected from standard stellar evolution theory, and they may affect the integrated spectra of their host clusters by contributing excess spectral energy in blue and ultraviolet bands. Many mechanisms, including single-star and binary models, have been proposed to account for the existence of BSs (see the review by Stryker 1993). At present, it is widely believed that more than one mechanism plays a role in the production of BSs in clusters and that binaries are important or even dominant for the production of BSs in open clusters and in the field (Lanzoni et al. 2007; Dalessandro et al. 2008; Sollima et al. 2008). Binaries may produce BSs through mass transfer, coalescence of the two components, binary-binary and binary-single star collisions. The collision of binarybinary or binary-single stars may lead binaries to be tighter or farther apart, which is relevant for the dynamics and the environment in the host cluster. In this contribution, we are only concerned with BSs resulting from the evolution of primordial binaries, i.e., mass transfer and coalescence of the two components.

\section{Evolutionary channels}

Before we describe the details of the evolutionary channels to BSs relevant to binary evolution, we first introduce the critical mass ratio, $q_{\mathrm{c}}$, for dynamically unstable mass transfer, which is crucial to determine the fate of a binary during mass transfer. The value of $q_{\mathrm{c}}$ differs for different evolutionary stages of the primary at the onset of mass transfer, and it has been well studied through polytropic models (Hjellming \& Webbink 1987; Han et al. 2001) and detailed binary evolution (Han et al. 2002; Chen \& Han 2008a). Figure 1 shows two examples of these studies. We see obvious differences for $q_{\mathrm{c}}$ between polytropic models and detailed binary evolution, which in turn lead to differences in the products, including BSs, after mass transfer. 

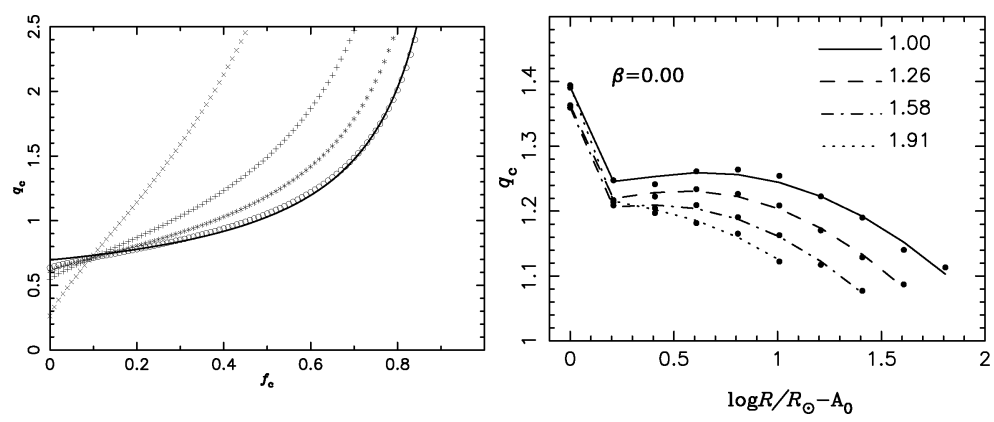

Figure 1. Critical mass ratio, $q_{\mathrm{c}}$, for dynamically unstable mass transfer. (left) Polytropic model. $f_{\mathrm{c}}$ is the core mass fraction of the mass donor. The crosses, pluses, asterisks and circles are for mass-transfer efficiencies $\beta=0.25,0.5,0.75$ and 1.0, respectively. The lost mass is assumed to carry away the same specific angular momentum as pertains to the mass donor. The solid line is from Webbink (1988) for conservative mass transfer (see also Han et al. 2001). (right) Detailed binary evolution for low-mass binaries between a first-ascent giant-branch and a main-sequence star. The giant star has a mass of $1.00,1.26,1.60$ and $1.90 M_{\odot}$, respectively. The lines are from the fitting formulae of equations (4) to (6) in Chen \& Han (2008a).

\subsection{Mass transfer}

In general, if the mass ratio $q=M_{1} / M_{2}$ (donor/accretor mass) at the onset of mass transfer is lower than $q_{\mathrm{c}}$, mass transfer is stable, and the accretor evolves upwards along the main sequence in response to accretion, if it is a main-sequence star, and becomes a BS when it is more massive than the turnoff of the host cluster.

This channel may produce binary BSs with various orbital periods (Chen \& Han 2004, 2008a). If the primary is on the main sequence or in the Hertzsprung gap at the onset of mass transfer, the products have short or relatively short orbital periods. These include Algol systems, which are still in a slow stage of mass transfer. Mass transfer between giant stars and main-sequence companions may produce long-orbital-period BSs. The value of $q_{\mathrm{c}}$ here significantly affects the BS number and orbital periods (Chen \& Han 2008a).

Since the accreted material may be originating from the nuclear region of the mass donor, it is rich in helium compared to the accretor's surface and has a higher mean molecular weight, resulting in secular instability during or after accretion. Thermohaline mixing will occur in this case. This mixing was once believed to cause the surfaceabundance abnormality of the accretor to be invisible. However, the study of Chen \& Han (2004) showed no distinction in surface composition between the models with and without thermohaline mixing during mass transfer, although their evolutionary tracks diverge. After mass transfer, CNO abundance abnormalities may exist for about $10^{8} \mathrm{yr}$, comparable to the lifetime of a typical BS. Thus, we could observe CNO abundance abnormalities in BSs this way.

\subsection{Coalescence of contact binaries}

During mass transfer, the accretor likely fills its Roche lobe and the system becomes a contact binary. This contact binary eventually coalesces into a single star (Webbink 1976; Eggleton 2000; Li et al. 2005). If both components are on the main sequence, their remnant is also a main-sequence star and evolves similarly to a normal star with that mass. So, the remnant may be a BS if it is more massive than the turnoff.

By assuming that the matter from the secondary mixes homogeneously with the envelope of the primary and that no mass is lost from the system during the merger process, Chen \& Han (2008b) constructed mergers of contact binaries and studied their characteristics. Their study shows that some mergers are located on the left of the zero-age 
main sequence defined by normal surface composition (i.e., helium content $Y=0.28$ and metallicity $Z=0.02$ for Population I) on a colour-magnitude diagram, because of enhanced surface-helium content. In addition, the central hydrogen content of the mergers is independent of mass. Thus, the concentration towards the blue side of the main sequence with decreasing mass predicted by Sandquist \& Shetrone (2003), does not appear in their models. In fact, there is no evidence for such a concentration from observations.

In old clusters, angular-momentum loss (AML) of low-mass binaries induced by magnetic braking is a main factor leading to contact binaries and final merging. A simple estimation (Chen \& Han 2008b) shows that, in old clusters, BSs resulting from AML are much more numerous than those originating from evolutionary effects only, indicating that AML in low-mass binaries makes a major contribution to BSs in old clusters such as NGC 188, NGC 2682, etc. In clusters of intermediate age, e.g., in NGC 2660, the models of Chen \& Han (2008b) can account for several BSs, although in the most likely region in the colour-magnitude diagram of NGC 2660 no BSs have as yet been observed. About $0.5 \mathrm{M}_{\odot}$ of mass loss in the merger process is necessary to resolve this conflict.

\subsection{Coalescence due to dynamically unstable mass transfer}

If the mass ratio, $q$, is greater than $q_{\mathrm{c}}$, mass transfer is dynamically unstable, and a common envelope (CE) is formed. The CE may be ejected if the orbital energy deposited into the envelope overcomes its binding energy, or else the binary will merge into a single star. If the two components are main-sequence stars, the remnant of coalescence will be on the main sequence, and it becomes a BS if its mass is higher than the turn-off mass of the host cluster.

In this case, the core of the secondary spirals in quickly and remains in the centre of the merger. The merger then has a chemical composition similar to that of the primary, resembling the result of smoothed-particle-hydrodynamics calculations (Lombardi et al 1996; Sills \& Lombardi 1997; Sills et al. 2001). Binary coalescence of a contact binary or dynamically unstable mass transfer is a popular hypothesis for the formation of single BSs (Mateo et al. 1990; Pols \& Marinus 1994; Andronov et al. 2006; Chen \& Han 2008b).

\section{Binary population synthesis}

We performed five sets of simulations for a Population I composition $(X=0.70, Y=$ 0.28 and $Z=0.02$ ) to systematically investigate BS formation from primordial binary evolution. The age ranges from 0.1 to 20 Gyr. The mass-transfer efficiency, $\beta$, defined as the mass fraction of the matter lost from the primary and accreted by the secondary, is an important parameter affecting the final results. Generally, we set $\beta=1$ when the mass donor is on the main sequence and $\beta=0.5$ otherwise. However this value is very uncertain, and it is likely higher than 0.5 when the mass donor is in the Hertzsprung gap and less than 0.5 when the mass donor is on the first giant branch or the AGB. We therefore also studied the case of $\beta=1$, when mass transfer begins in the Hertzsprung gap (Chen \& Han 2009).

\subsection{Distribution in colour-magnitude diagrams}

Figure 2 shows colour-magnitude diagrams of a population with an age of 4.3 Gyr for various $\beta$ values when the primary is in the Hertzsprung gap at the onset of Rochelobe overflow. We see that BSs from $\beta=1$ may be more massive than those for $\beta=0.5$, indicating that a high value of $\beta$ may produce BSs far away from the turnoff. In particular, we may obtain BSs with masses higher than twice the turnoff mass, even though these objects have very short lifetimes. It is appropriate to assume a high value of $\beta$ for old 


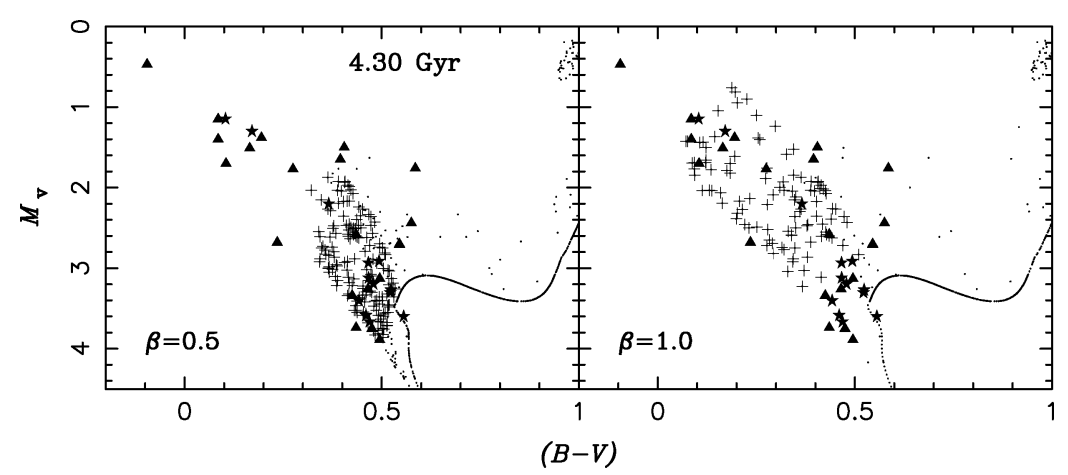

Figure 2. Colour-magnitude diagrams for a 4.3 Gyr-old population. Here, $\beta$ is the mass fraction of the matter lost from the primary and accreted by the secondary when the primary is in the Hertzsprung gap at the onset of mass transfer. The crosses and stars are for BSs from mass transfer and binary coalescence, respectively, and the small dots are for other objects in the population. The triangles are observed BSs in M67 from Sandquist \& Shetrone (2003).

clusters, since the binaries contributing to BSs are less massive than those in young clusters.

We also see in the figure that many BSs are below but bluer than the turnoff. They may extend into the region 1 mag below the turnoff. These objects are mainly formed through mass transfer and binary coalescence of dynamically unstable mass transfer. They are less evolved than the turnoff and may contribute more to the flux in the $V$ band in the subsequent evolution. Most BSs from mass transfer and binary coalescence of dynamically unstable mass transfer are within 1.5 mag of the turnoff, while some from binary coalescence of contact binaries may have magnitudes about 2.3 mag above the turnoff.

\subsection{The specific frequency}

The BS number from the simulations, $N_{\mathrm{BS}}$, depends slightly on the population's age, while the specific frequency, $\log F_{\mathrm{BSS}}\left(\equiv \log \left(N_{\mathrm{BS}} / N_{2}\right)\right.$, where $N_{2}$ is the number of stars within 2 mag below the main-sequence turnoff), depends heavily on age due to the related increase of $N_{2}$. In all of the five sets, $\log F_{\mathrm{BSS}}$ first decreases with time and then increases when the age becomes older than 10 Gyr. The decrease of $\log F_{\mathrm{BSS}}$ before 1.5 Gyr comes from the increase of $N_{2}$. Subsequently, the number of potential binaries which may contribute to BSs decreases, leading to the formation of fewer BSs. On the other hand, $N_{2}$ continues to increase. Thus, $\log F_{\mathrm{BSS}}$ continues to decrease. Over time, the primaries in long-orbital-period binaries gradually enter the AGB phase, expand dramatically and some may fill their Roche lobe and start mass transfer. Due to strong stellar winds in the AGB phase, these mass donors at the onset of mass transfer are probably much less massive than before and this mass transfer is easily stabilized, resulting in some longorbital-period BSs. As a consequence, $\log F_{\mathrm{BSS}}$ begins to increase when their age is older than 10 Gyr. AML becomes more and more important for BS formation with time and exceeds that from primordial binary evolution when the age is older than 2.5 Gyr.

When we investigated the role of primordial binary evolution for BS formation in Galactic open clusters, we found a serious problem that the BS specific frequency obtained in our simulations is much lower (only about 20\%) than observed, which may result from the following aspects. (i) Observational errors, such as $N_{2}$ counted in clusters, the cluster ages and the BS sample, etc. For example, the study of Carraro (2008) showed that a large fraction of earlier BSs are actually field stars. (ii) The adopted Monte Carlo simulation 


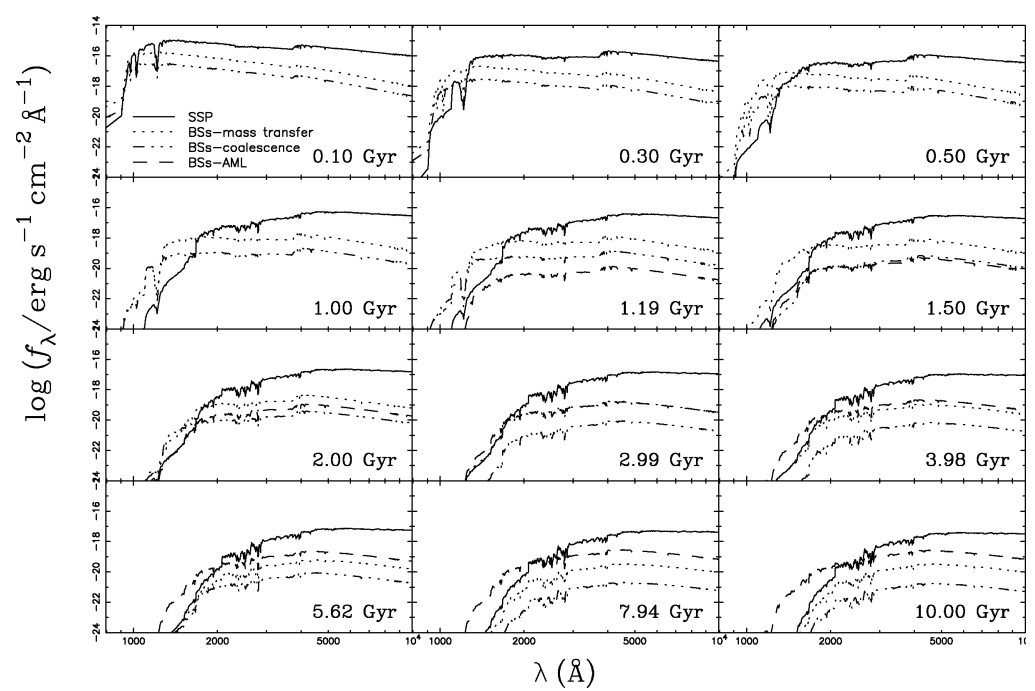

Figure 3. Integrated rest-frame intrinsic spectral-energy distributions for a stellar population with a mass of $1 \mathrm{M}_{\odot}$ at a distance of $10 \mathrm{kpc}$ with $\beta=0.5$ when the primary is in the Hertzsprung gap at the onset of mass transfer. The solid lines are for the SSP (a population without binary interactions) and the other lines are the contributions of BSs from different evolutionary channels.

parameters, including the initial mass function, distributions of the initial ratio and the initial separation. All these parameters in our simulation are for field stars and it is very likely that they are different in clusters. For instance, recent studies show that the initial mass function might be quite different for disk and halo stars in the Galaxy (Pols et al. 2008). (iii) Other channels, e.g., a more recent era of star formation and dynamical interaction may produce BSs in open clusters in addition to primordial binary evolution and AML.

\subsection{Contribution to the integrated spectral-energy distributions}

We plotted the integrated spectral-energy distributions (ISEDs) of a population for various cases in Figure 3, where SSP refers to a population without binary interaction. This figure shows that BSs resulting from binary evolution are dominant contributors to the ISED in ultraviolet and blue bands for ages between 0.3 and 2.0 Gyr. The BSs and SSP have comparable energy in the ultraviolet and blue between 2 and 4 Gyr. The contribution from AML becomes more important with time, and exceeds that from primordial binary evolution for a population older than $\sim 3$ Gyr. Thus, primordial binaries are important contributors to BSs over the whole age range. Since BSs from a high $\beta$ have higher masses, their contributions to the ISED become more important in ultraviolet and blue bands (see figure 5 in Chen \& Han 2009).

\subsection{Influence on colours and ages}

Obviously, the excess spectral energy due to BSs in ultraviolet and blue bands inevitably results in some changes in colours involving these bands. The results are shown in Figure 4 , from which we see that the colours are affected from about 0.32 Gyr (all five colours shown in this figure) to older than 10 Gyr (i.e., F185W-F336W and F218W-F336W), and the maximum difference may be up to 1.5 mag for F170W-F336W. 


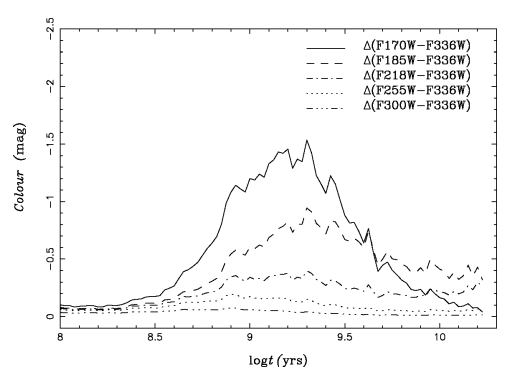

Figure 4. Colour differences versus age for a population with and without BSs.

\section{Conclusions}

We have summarized the links between binary evolution and BSs, and the relevant BS characteristics. We also showed binary population synthesis results of BSs from primordial binary evolution, such as their distribution in the colour-magnitude diagram, their contribution to the ISED, the specific frequency and their influence on colours.

\section{Acknowledgements}

This work was in part supported by the Chinese National Science Foundation (grants 10973036, 10603013, 10821061 and 2007CB815406) and the Yunnan National Science Foundation (grant 08YJ041001).

\section{References}

Andronov, N., Pinsonneault, M. H., \& Terndrup, D. M. 2006, ApJ, 646, 1160

Carraro, G., 2008, A\&A, 482, 777

Chen, X. \& Han, Z. 2004, MNRAS, 355, 1182

Chen, X. \& Han, Z. 2008a, MNRAS, 387, 1416

Chen, X. \& Han, Z. 2008b, MNRAS, 384, 1263

Chen, X. \& Han, Z. 2009, MNRAS, 395, 1822

Dalessandro, E., Lanzoni, B., Ferraro, F. R., Rood, R. T., Milone, A., Piotto, G., \& Valenti, E. 2008, ApJ, 677, 1069

Eggleton, P. P. 2000, NewAR, 44, 111

Han, Z., Eggleton, P. P., Podsiadlowski, P., Tout C. A., \& Webbink, R. F. 2001, ASP Conf. Ser., 229, 205

Han, Z., Podsiadlowski, P., Maxted, P. F. L., Marsh, T. R., \& Ivanova, N. 2002, MNRAS, 336, 449

Hjellming, M. S. \& Webbink, R. F. 1987, ApJ, 318, 794

Lanzoni, B., Dalessandro, E., Perina, S., Ferraro, F. R., Rood, R. T., \& Sollima, A. 2007, ApJ, 670,1065

Li, L., Han, Z., \& Zhang, F. 2005, MNRAS, 360, 272

Lombardi Jr., J. C., Rasio, F. A., \& Shapiro, S. L. 1996, ApJ, 468, 797

Mateo, M., Harris, H., Nemec, J., \& Olszewski, E. 1990, AJ, 100, 469

Pols, O. R., Izzard, R. G., Lugaro, M., \& de Mink, S. E. 2008, IAU Symp., 252, 383

Pols, O. R. \& Marnus, M. 1994, A\& A, 288, 475

Sandquist, E. L. \& Shetrone, M. D. 2003, AJ, 125, 2187

Sills, A. \& Lombardi Jr., J. C. 1997, ApJ, 105, 1081

Sills, A., et al. 2001, ApJ, 105, 1081

Sollima, A., Lanzoni, B., Beccari, G., Ferraro, F. R., \& Fusi Pecci, F. 2008, A $\triangleleft A, 481,701$

Stryker, L. L. 1993, PASP, 105, 1081

Webbink, R. F. 1976, ApJ, 209, 829

Webbink, R. F. 1988, in: Mikolajewska, J., Friedjung, M., Kenyon, S. J., \& Viotti, R., The Symbiotic Phenomenon, p. 311 (Dordrecht: Kluwer) 